4

$$
5
$$

$6 \quad{ }^{1}$ Drug Discovery and Safety, Leiden Academic Centre for Drug Research, Einsteinweg

7 55, Leiden, The Netherlands

$8{ }^{2}$ School of Electrics and Information Engineering, Xi' an Jiaotong University, 28

9 XianningW Rd, Xi'an, China

$10 \quad{ }^{3}$ Janssen Pharmaceutica NV, Turnhoutseweg 30, B-2340, Beerse, Belgium

\section{DrugEx v3: Scaffold-Constrained Drug Design with Graph Transformer-based Reinforcement Learning}

3 Xuhan Liu ${ }^{1}$, Kai Ye ${ }^{2}$, Herman W. T. van Vlijmen ${ }^{1,3}$, Adriaan P. IJzerman ${ }^{1}$, Gerard J. P. van Westen ${ }^{1, *}$

"To whom correspondence should be addressed: Gerard J. P. van Westen, Drug Discovery and Safety, Leiden Academic Centre for Drug Research, Einsteinweg 55, Leiden, The Netherlands. Tel: +31-71-527-3511. Email: gerard@lacdr.leidenuniv.nl.

Email Address of other authors: (1) Xuhan Liu: x.liu@lacdr.leidenuniv.nl; (2) Kai Ye: 17

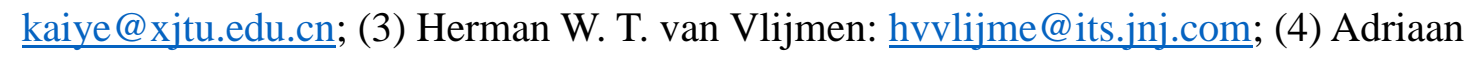
P. IJzerman: ijzerman@lacdr.leidenuniv.nl. 


\section{Abstract}

Due to the large drug-like chemical space available to search for feasible drug-like molecules, rational drug design often starts from specific scaffolds to which side chains/substituents are added or modified. With the rapid growth of the application of deep learning in drug discovery, a variety of effective approaches have been developed for de novo drug design. In previous work, we proposed a method named DrugEx, which can be applied in polypharmacology based on multi-objective deep reinforcement learning. However, the previous version is trained under fixed objectives similar to other known methods and does not allow users to input any prior information (i.e. a desired scaffold). In order to improve the general applicability, we updated DrugEx to design drug molecules based on scaffolds which consist of multiple fragments provided by users. In this work, the Transformer model was employed to generate molecular structures. The Transformer is a multi-head self-attention deep learning model containing an encoder to receive scaffolds as input and a decoder to generate molecules as output. In order to deal with the graph representation of molecules we proposed a novel positional encoding for each atom and bond based on an adjacency matrix to extend the architecture of the Transformer. Each molecule was generated by growing and connecting procedures for the fragments in the given scaffold that were unified into one model. Moreover, we trained this generator under a reinforcement learning framework to increase the number of desired ligands. As a proof of concept, our proposed method was applied to design ligands for the adenosine $A_{2 A}$ receptor $\left(\mathrm{A}_{2 \mathrm{~A}} \mathrm{AR}\right)$ and compared with SMILES-based methods. The results demonstrated the effectiveness of our method in that $100 \%$ of the generated molecules are valid and most of them had a high predicted affinity value towards $A_{2 A} A R$ with given scaffolds.

Keywords: deep learning, reinforcement learning, policy gradient, drug design, Transformer, multi-objective optimization 


\section{Introduction}

Due to the size of drug-like chemical space (i.e. estimated at $10^{33}-10^{60}$ organic molecules) ${ }^{1}$ it is impossible to screen every corner of it to discover optimal drug candidates. Commonly, the specific scaffolds derived from endogenous substances, high throughput screening, or a phenotypic assay ${ }^{2}$ are taken as a starting point to design analogs while side chains/substituents are added or modified ${ }^{3}$. These fragments are used as "building blocks" to develop drug leads with e.g. combinatorial chemistry such as growing, linking, and merging ${ }^{4}$. After a promising drug lead has been discovered it is further optimized by modifying side chains to improve potency towards the relevant targets, selectivity over off-targets, and physicochemical properties which in turn can improve safety and tolerability ${ }^{5}$.

In scaffold-based rational drug design, it is generally accepted that a chemical space consisting of $10^{9}$ diverse molecules can be sampled with only $10^{3}$ fragments ${ }^{6}$. For instance, one well known class of drug targets are G Protein-coupled receptors (GPCRS), a family via which approximately $35 \%$ of drug exert their effect ${ }^{7}$. The adenosine receptors (ARs) form a family within rhodopsin-like GPCRs and include four subtypes $\left(\mathrm{A}_{1}, \mathrm{~A}_{2 \mathrm{~A}}, \mathrm{~A}_{2 \mathrm{~B}}\right.$ and $\left.\mathrm{A}_{3}\right)$. Each of them has a unique pharmacological profile, tissue distribution, and effector coupling 8, 9. ARs are ubiquitously distributed throughout the human tissues, and involved in many biological processes and diseases ${ }^{10}$. As adenosine is the endogenous agonist of ARs, a number of known ligands of the ARs are adenosine analogs and have a common scaffold. Examples include purines, xanthines, triazines, pyrimidines, and the inclusion of a ribose moiety ${ }^{11}$. In this work, we aim to design novel ligands for this family of receptors using a deep learning-based drug design method. 
Deep learning based methods have been gaining ground in computational drug discovery, including de novo design, based on rapid developments over the last decade ${ }^{12}$. Deep learning has achieved breakthroughs in visual recognition, natural language processing, and other data-rich fields ${ }^{13}$. For distribution-directed issues, GomezBombarelli et al. implemented variational autoencoders (VAE) to map molecules into a latent space where each point can also be decoded into unique molecules inversely ${ }^{14}$. They used recurrent neural networks (RNNs) to successfully learn SMILES (simplified molecular-input line-entry system) grammar and construct a distribution of molecular libraries ${ }^{15}$. For goal-directed issues, Sanchez-Lengeling et al. combined reinforcement learning and generative adversarial networks (GANs) to develop an approach named ORGANIC to design active compounds for a given target ${ }^{16}$. Olivecrona et al. proposed the REINVENT algorithm which updated the reinforcement learning with a Bayesian approach and combined RNNs to generate SMILES-based desired molecules ${ }^{17,} 18$. Moreover, Lim et al. proposed a method for scaffold-based molecular design with a graph generative model ${ }^{19}$. Li et al. also used deep learning to develop a tool named DeepScaffold for this issue ${ }^{20}$. Arús-Pous et al. employed RNNs to develop a SMILESbased scaffold decorator for de novo drug design ${ }^{21}$. Yang et al. used the Transformer model $^{22}$ to develop a tool named SyntaLinker for automatic fragment linking ${ }^{23}$. Here we continue to address on this issue further with different molecular representations and deep learning architectures.

In previous studies we investigated the performance of RNNs and proposed a method named DrugEx by integrating reinforcement learning to balance distribution-directed and goal-directed tasks ${ }^{24}$. Furthermore, we updated DrugEx with multi-objective reinforcement learning and applied it in polypharmacology ${ }^{25}$. However, the welltrained model cannot receive any input data from users and can only reflect the distribution of the desired molecules with fixed conditions. If the objectives are changed, the model needs to be trained again. In this work, we compared different end-to-end deep learning methods to update the DrugEx model to allow users to provide prior information, e.g. fragments that should occur in the generated molecules. Based on the 
105 extensive experience in our group with the $\mathrm{A}_{2 \mathrm{~A}} \mathrm{AR}$, we continue to take this target as an 106 example to evaluate the performance of our proposed methods. In the following context, 107 we will discuss the case of scaffold-constrained drug design, i.e. the model takes 108 scaffolds composed of multiple fragments as input to generate desired molecules which 109 are predicted to be active to $\mathrm{A}_{2 \mathrm{~A} A R}$. All python code for this study is freely available 110 at http://gitlab.com/XuhanLiu/DrugEx. 


\section{Materials and Methods}

\section{Data source}

The ChEMBL set was reused from our work on DrugEx $v 2{ }^{25}$. This set consisted of small molecule compounds downloaded from ChEMBL using a SMILES notation (version 27$)^{26}$. There were $\sim 1.7$ million molecules remained for model pre-training after data preprocessing implemented by RDKit. Preprocessing included neutralizing charges, removing metals and small fragments. In addition, 10,828 ligands and bioactivity data were extracted from ChEMBL to construct the LIGAND set, containing structures and activities from bioassays towards the four human adenosine receptors. The LIGAND set was used for fine-tuning the generative model. Molecules with annotated $\mathrm{A}_{2 \mathrm{~A}} \mathrm{AR}$ activity were used to train a bioactivity prediction model. If multiple measurements for the same ligand existed, the average $\mathrm{pChEMBL}$ value ( $\mathrm{pX}$, including pKi, pKd, pIC50 or pEC50) was calculated and duplicate items were removed. In order to judge if the molecule is desired or not, the threshold of affinity was defined as $\mathrm{pX}=$ 6.5 to predict if the compound was active $(>=6.5)$ or inactive $(<6.5)$.

The dataset was constructed with an input-output pair for each data point. Each molecule was decomposed into a batch of fragments with the BRICS method ${ }^{27}$ in RDKit (Fig 1A). If a molecule contained more than four leaf fragments, the smaller fragments were ignored and a maximum of four larger fragments were reserved to be randomly combined at one time. Their SMILES sequences were joined with '.' as input data which were paired with the full SMILES of molecules. Here, the scaffold was defined as the combination of different fragments which can be either continuous (linked) or discrete (separated). The resulting scaffold-molecule pairs formed the input and output data (Fig 1B). After completion of construction of the data pairs the set was split into a training set and test set with the ratio 9:1 based on the input scaffolds. The resulting ChEMBL set contained 10,418,681 and 1,083,271 pairs for training and test set, respectively. The LIGAND set contained 61,413 pairs in the training set and 7,525 pairs in the test set. 
A

Leaf Fragments
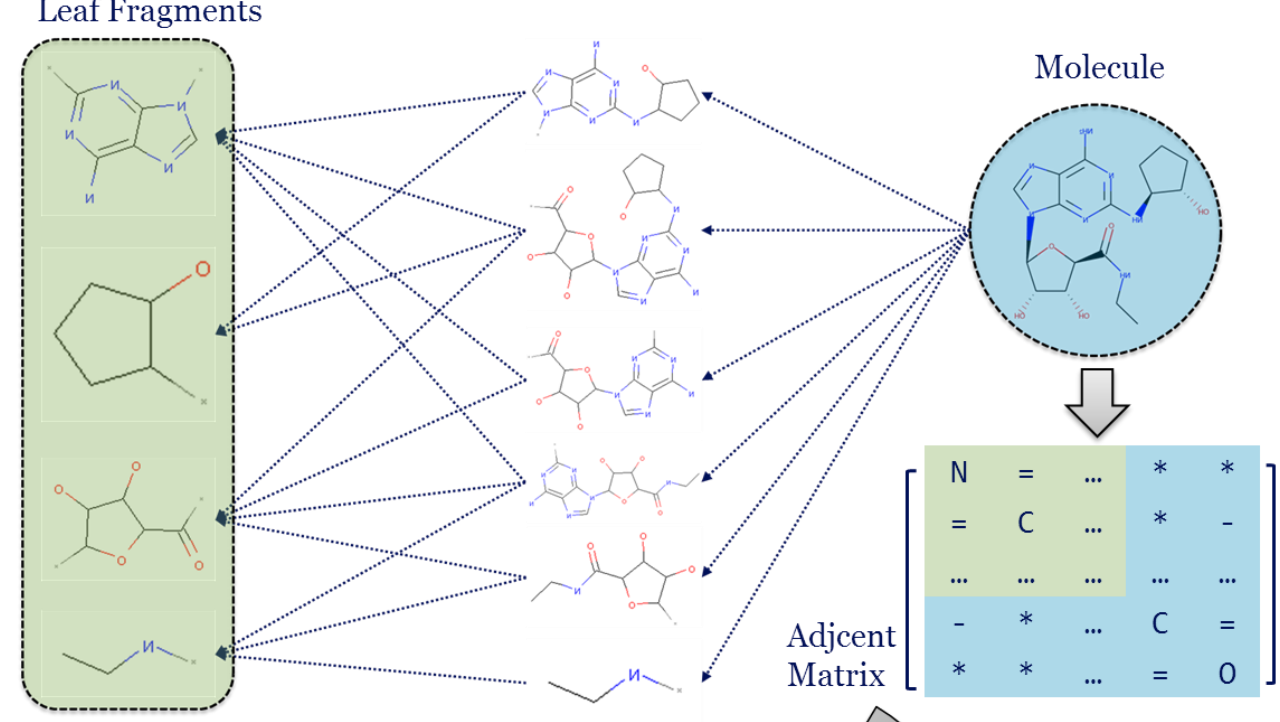

Input (Scaffold)

Output (Molecule)

\begin{tabular}{|c|c|}
\hline NCC.C1CCCC10 & $\operatorname{CCNC}(=0) \operatorname{C} 10 \mathrm{C}(\mathrm{n} 2 \mathrm{cnc} 3 \mathrm{C}(\mathrm{N}) \mathrm{nc} . .$. \\
\hline$C(=0) C 10 C(n 2 c n c 3 \ldots$ & $\operatorname{CCNC}(=0) \operatorname{C} 10 \mathrm{C}(n 2 \mathrm{cnc} 3 \mathrm{C}(\mathrm{N}) \mathrm{nc} . .$. \\
\hline C1CCCC10.C (=0)C1... & $\operatorname{CCNC}(=0) \operatorname{C} 10 \mathrm{C}(n 2 \mathrm{cnc} 3 \mathrm{C}(\mathrm{N}) \mathrm{nc} . .$. \\
\hline$C(=0) C 10 C(n 2 c n c 3 \ldots$ & $\operatorname{CCNC}(=0) \operatorname{C} 10 \mathrm{C}(\mathrm{n} 2 \mathrm{cnc} 3 \mathrm{C}(\mathrm{N}) \mathrm{nc} . .$. \\
\hline$C(=0) C 10 C C(0) C 10$ & $\operatorname{CCNC}(=0) \operatorname{C10C}(n 2 \operatorname{cnc} 3 c(N) n c . .$. \\
\hline$c 1 n c(N) c 2 n c n c 2 n 1$ & $\operatorname{CCNC}(=0) \operatorname{C10C}(n 2 \operatorname{cnc} 3 c(N) n c . .$. \\
\hline
\end{tabular}

…... ......

C Graph Matrix

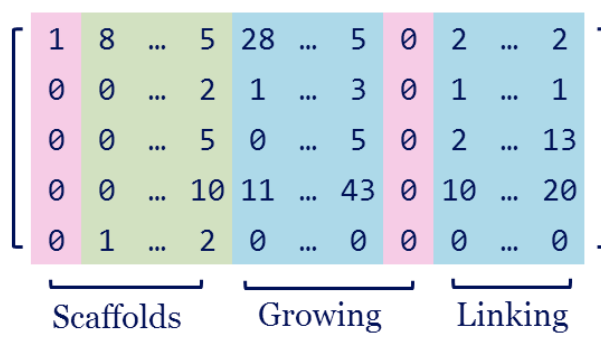

Fig. 1: scaffold-molecule pair dataset construction. (A) Each molecule in the dataset is decomposed hierarchically into a series of fragments with the BRICS algorithm. (B) Subsequently data pairs between input and output are created. Combinations of leaf fragments form the scaffold as input, while the whole molecule becomes the output. Each token in the SMILES sequences is separated by different colors. (C) After conversion to the adjacency matrix, each molecule was represented as a graph matrix. The graph matrix contains five rows, standing for the atom, bond, previous and current positions, and fragment index. The columns are composed with three parts to store the information of the scaffold, the growing section and the linking section. (D) All tokens are collected to construct the vocabularies for SMILES-based and graph-based generators, respectively. (E) An example of the input and output matrices for the SMILES representation of scaffolds and molecules 
150

In this study we tested two different molecular representations: SMILES and graph. For SMILES representations each scaffold-molecule pair was transformed into two SMILES sequences which were then split into different tokens to denote atoms, bonds, or other tokens for grammar control (e.g. parentheses or numbers). All of these tokens were put together to form a vocabulary which recorded the index of each token (Fig. 1D). Here, we used the same conversion procedure and vocabulary as in DrugEx v2 25 . In addition, a start token (GO) was put at the beginning of a batch of data as input and an end token (END) at the end of the same batch of data as output. After sequence padding with a blank token at empty positions, each SMILES sequence was rewritten as a series of token indices with a fixed length. Subsequently all of these sequences for both scaffolds and molecules were concatenated to construct the input and output matrix (Fig. 1E).

For the graph representation each molecule was represented as a five-row matrix, in which the first two rows stand for the index of the atom and bond types, respectively. The third and fourth rows represent the position of previous and current atoms connected by a bond (Fig. 1C). The columns of this matrix contain three sections to store the scaffold, growing part, and linking part. The scaffold section began with a start token in the first row and the last row was labelled with the index of each scaffold starting from one. The scaffolds of each molecule are put in the beginning of the matrix, followed by the growing part for the scaffold, and the last part is the connecting bond between these growing fragments with single bonds. For the growing and linking sections the last row was always zero and these two sections were separated by the column of the end token. It is worth noticing that the last row was not directly involved in the training process. The vocabulary for graph representation was different from the SMILES representation, contains 38 atom types (Table S1), and four bond types (single, double, triple bonds and no bond). For each column, If an atom is the first occurrence in a given scaffold the type of the bond will be empty (indexed as 0 with token '*'). In addition, if the atom at the current position has occurred in the matrix, the type of the 
atom in this column will be empty. In order to grasp more details of the graph representation, we also provided the pseudocode for encoding (Table S2) and decoding (Table S3).

\section{End-to-End Deep learning}

In this work, we compared three different sequential end-to-end DL architectures to deal with different molecular representations of either graph or SMILES (Fig. 2). These methods included: (A) a Graph Transformer, (B) an LSTM-based encoder-decoder model (LSTM-BASE), (C) an LSTM-based encoder-decoder model with an attention mechanism (LSTM+ATTN) and (D) a Sequential Transformer model. All of these DL models were constructed with PyTorch ${ }^{28}$.

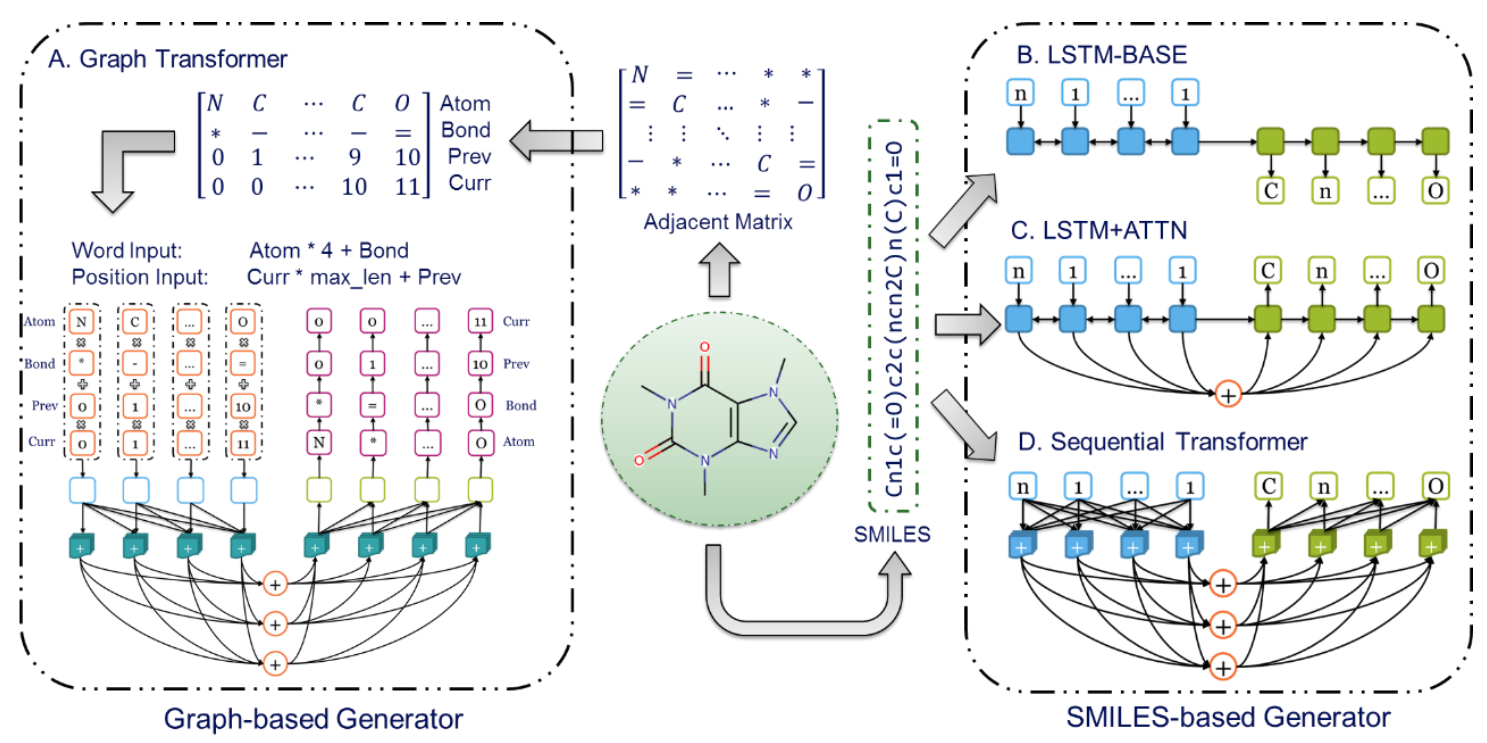

Fig. 2: Architectures of four different end-to-end deep learning models: (A) The Graph Transformer; (B) The LSTM-based encoder-decoder model (LSTM-BASE); (C) The LSTM-based encoder-decoder model with attention mechanisms (LSTM+ATTN);

(D) The sequential Transformer model. The Graph Transformer accepts a graph representation as input and SMILES sequences are taken as input for the other three models.

For the SMILES representation based models three different types were constructed as follows (Fig. 2, right). The encoder and decoder in the LSTM-BASE model (Fig. 2B) 
had the same architectures, containing one embedding layer, three recurrent layers, and one output layer (as used in DrugEx v2). The number of neurons in the embedding and hidden layers were 128 and 512, respectively. The hidden states of the recurrent layer in the encoder are directly sent to the decoder as the initial states. On the basis of the LSTM-BASE model an attention layer was added between the encoder and decoder to form the LSTM+ATTN model (Fig. 2C). The attention layer calculates the weight for each position of the input sequence to determine which position the decoder needs to focus on during the decoding process. For each step the weighted sums of the output calculated by the encoder are combined with the output of the embedding layer in the decoder to form the input for the recurrent layers. The output of the recurrent layers is dealt with by the output layer to generate the probability distribution of tokens in the vocabulary in both of these two models.

The sequential Transformer has a distinct architecture compared to the LSTM+ATTN model although it also exploits an attention mechanism. For the embedding layers "position encodings" are added into the typical embedding structure as the first layer of the encoder and decoder. This ensures that the model no longer needs to encode the input sequence token by token but can process all tokens in parallel. For the position embedding, sine and cosine functions are used to define its formula as follows:

$$
\begin{gathered}
P E_{(p, 2 i)}=\sin \left(p o s / 10000^{2 i / d_{m}}\right) \\
P E_{(p, 2 i+1)}=\cos \left(p o s / 10000^{2 i / d_{m}}\right)
\end{gathered}
$$

where $P E(p, i)$ is the $i^{t h}$ dimension of the position encoding at position $p$. It has the same dimension $d_{m}=512$ as the typical embedding vectors so that the two can be summed.

In addition, self-attention is used in the hidden layers to cope with long-range dependencies. For each hidden layer in the encoder, it employs a residual connection around a multi-head self-attention sublayer and feed-forward sublayer followed by layer normalization. Besides these two sublayers in the decoder a third sublayer with multi-head attention is inserted to capture the information from output of the encoder. 
This self-attention mechanism is defined as the scaled dot-product attention with three 231 vectors: queries $(Q)$, keys $(K)$ and values $(V)$, of which the dimensions are $d_{q}, d_{k}, d_{v}$, respectively. The output matrix is computed as:

$$
\operatorname{Attention}(Q, K, V)=\operatorname{softmax}\left(\frac{Q K^{\top}}{\sqrt{d_{k}}}\right) V
$$

Instead of a single attention function, the Transformer adopts multi-head attention to combine information from different representations at different positions which is defined as:

$$
\operatorname{MultiHead}(Q, K, V)=\text { Concat }\left(\text { head }_{1}, \ldots, \text { head }_{h}\right) W^{O}
$$

where $h$ is the number of heads. For each head, the attention values were calculated by different and learned linear projections with $Q, K$ and $V$ as follows:

$$
\text { head }_{i}=\operatorname{Attention}\left(Q W_{i}^{Q}, K W_{i}^{K}, V W_{i}^{V}\right)
$$

where $W^{O}, W^{Q}, W^{K}$ and $W^{V}$ are metrics of learned weights and we set $h=8$ as the number of heads and $d_{k}=d_{v}=64$ in this work.

For the graph representation of the molecules we updated the sequential Transformer structure to propose a Graph Transformer (Fig. 2A). Similar to the sequential Transformer the Graph Transformer also requires the encodings of both word and position as the input. For the input word, the atom and bond cannot be processed simultaneously; therefore we combined the index of atom and bond together and defined it as follows:

$$
I=I_{\text {atom }} \times 4+I_{\text {bond }}
$$

The index of the input word $(I)$ for calculating word vectors is obtained by multiplying the atom index $\left(I_{\text {atom }}\right)$ by four (the total number of bond types defined) and subsequently add the bond index $\left(I_{b o n d}\right)$. Similarly, the position of each step cannot be used to calculate the position encoding directly. Faced with more complex data structure than sequential data, Dosovitskiy et al. proposed a new positional encoding scheme to define the position for each patch in image data for image recognition ${ }^{29}$. Inspired by their 
work the position encoding at each step was defined as:

$$
P=P_{\text {curr }} \times L_{\text {max }}+P_{\text {prev }}
$$

The input position $(P)$ for calculating the position encoding was obtained by multiplying the current position $\left(P_{\text {curr }}\right)$ by the max length $\left(L_{\max }\right)$ and then adding the previous position $\left(P_{\text {prev }}\right)$, which was then processed with the same positional encoding method as with the sequential Transformer. For the decoder, the hidden vector from the transformer was taken as the starting point to be decoded by a GRU-based recurrent layer; and the probability of atom, bond, previous and current position was decoded one by one sequentially.

When graph-based molecules are generated, the chemical valence rule is checked in every step. Invalid values of atom and bond types will be masked and an incorrect previous or current position will be removed ensuring the validity of all generated molecules. It is worth noticing that before being encoded, each molecule will be kekulized, meaning that the aromatic rings will be inferred to transform into either single or double bonds. The reason for this is that aromatic bonds interfere with the calculation of the valence value for each atom.

During the training process of SMILES-based models, a negative log likelihood function was used to construct the loss function to guarantee that the probability of the token at each step in the output sequence became large enough in the probability distribution of the vocabulary calculated by the deep learning model. In comparison, the loss function used by the Graph Transformer model also contains four parts for atom, bond, previous and current sites. Here the sum of these negative log probability values is minimized to optimize the parameters in the model. For this, the Adam algorithm was used for the optimization of the loss function. Here, the learning rate was set as $10^{-}$ ${ }^{4}$, the batch size was 256 , and training steps were set to 20 epochs for pre-training and 1,000 epochs for fine-tuning. 


\section{Multi-objective optimization}

In order to combine multiple objectives we exploited a Pareto-based ranking algorithm with GPU acceleration as mentioned in DrugEx v2 ${ }^{25}$. Given two solutions $m_{1}$ and $m_{2}$ with their scores $\left(x_{1}, x_{2}, \ldots, x_{n}\right)$ and $\left(y_{1}, y_{2}, \ldots, y_{n}\right)$, then $m_{1}$ is said to Pareto dominate $m_{2}$ if and only if:

$$
\forall \mathrm{j} \in\{1, \ldots, \mathrm{n}\}: x_{j} \geq y_{j} \text { and } \exists \mathrm{j} \in\{1, \ldots, \mathrm{n}\}: x_{j}>y_{j}
$$

otherwise, $m_{1}$ and $m_{2}$ are non-dominated with each other. After the dominance between all pair of solutions being determined, the non-dominated scoring algorithm is exploited to obtain a rank of Pareto frontiers which consist of a set of solutions. After obtaining frontiers between dominant solutions, molecules were ranked based on the average Tanimoto-distance to other molecules instead of the commonly used crowding distance in the same frontier. Subsequently molecules with smaller average distances were ranked on the top. The final reward $R^{*}$ is defined as:

$$
R^{*}= \begin{cases}0.5+\frac{k-N_{\text {undesired }}}{2 N_{\text {desired }}}, & \text { if desired } \\ \frac{k}{2 N_{\text {undesired }}}, & \text { if undesired }\end{cases}
$$

here $k$ is the index of the solution in the Pareto rank. Rewards of undesired and desired solutions will be evenly distributed in $(0,0.5]$ and $(0.5,0.1]$, respectively.

In this work, we took two objectives into consideration: 1) the QED score ${ }^{30}$ as implemented by RDKit (from 0 to 1 ) to evaluate the drug-likeness of each molecule (a larger value means more drug-like) ; 2) an affinity score towards the $\mathrm{A}_{2 \mathrm{~A}} \mathrm{AR}$ which was implemented by a random forest regression model with Scikit-Learn ${ }^{31}$ like in DrugEx $v 2{ }^{25}$. The input descriptors consisted of 2048D ECFP6 fingerprints and 19D physicochemical descriptors (PhysChem). PhysChem included: molecular weight, logP, number of $\mathrm{H}$ bond acceptors and donors, number of rotatable bonds, number of amide bonds, number of bridge head atoms, number of hetero atoms, number of spiro atoms, number of heavy atoms, the fraction of SP3 hybridized carbon atoms, number of aliphatic rings, number of saturated rings, number of total rings, number of aromatic rings, number of heterocycles, number of valence electrons, polar surface area, and 
Wildman-Crippen MR value. Again it was determined if generated molecules are desired based on the Affinity score (larger than the threshold =6.5). In addition, the SA score was also exploited an independent measurement to evaluate the synthesizability of generated molecules, which is also calculated by RDKit ${ }^{32}$.

\section{Reinforcement learning}

In this work we constructed a reinforcement learning framework based on the interplay between a Graph Transformer (agent) and two scoring functions (environment). A policy gradient method was implemented to train the reinforcement learning model, the objective function is designated as follows:

$$
J(\theta)=\mathbb{E}\left[R^{*}\left(y_{1: T}\right) \mid \theta\right]=\sum_{t=1}^{T} \log G\left(y_{t} \mid y_{1: t-1}\right) \cdot R^{*}\left(y_{1: T}\right)
$$

For each step $t$ during the generation process the generator $(G)$ determines the probability of each token $\left(y_{t}\right)$ from the vocabulary to be chosen based on the generated sequence in previous steps $\left(y_{1: t-1}\right)$. In the sequence-based models $y_{t}$ can only be a token in the vocabulary to construct SMILES while it can be different type of atoms or bonds or the previous or current position in the graph-based model. The parameters in the objective function are updated by employing a policy gradient based on the expected end reward $\left(\mathrm{R}^{*}\right)$ received from the predictor. By maximizing this function the parameter $\theta$ in the generator can be optimized to ensure that the generator designs desired molecules which obtain a high reward score.

In order to improve the diversity and reliability of generated molecules, we implemented our exploration strategy for molecule generation during the training loops. In the training loop our generator is trained to produce the chemical space as defined by the target of interest. In this strategy there are two networks with the same architectures, an exploitation net $\left(G_{\theta}\right)$ and an exploration net $\left(G_{\varphi}\right) . G_{\varphi}$ did not need to be trained and its parameters are always fixed and it is based on the general drug-like chemical space for diverse targets obtained from ChEMBL. The parameters in $G_{\theta}$ on the other hand were updated for each epoch based on the policy gradient. Again an 
exploring rate $(\varepsilon)$ was defined with a range of $[0.0,1.0]$ to determine the percentage of scaffolds being randomly selected as input by $G_{\varphi}$ to generate molecules. Conversely $G_{\theta}$ generated molecules with other input scaffolds. After the training process was finished $G_{\varphi}$ was removed and only $G_{\theta}$ was left as the final model for molecule generation.

\section{Performance evaluation}

In order to evaluate the performance of the generators, four coefficients were calculated from the population of generated molecules (validity, accuracy, desirability, and uniqueness) which are defined as:

$$
\text { Validity }=\frac{N_{\text {valid }}}{N_{\text {total }}}
$$

$$
\text { Accuracy }=\frac{N_{\text {accurate }}}{N_{\text {total }}}
$$

$$
\text { Desirability }=\frac{N_{\text {desired }}}{N_{\text {total }}}
$$

$$
\text { Uniqueness }=\frac{N_{\text {unique }}}{N_{\text {total }}}
$$

here $N_{\text {total }}$ is the total number of molecules, $N_{\text {valid }}$ is the number of molecules parsed as valid SMILES sequences, $N_{\text {accurate }}$ is the number of molecules that contained all given scaffolds, $N_{\text {desired }}$ is the number of desired molecules that reach all required objectives, and $N_{\text {unique }}$ is the number of molecules which are different from others in the dataset.

To measure molecular diversity, we adopted the Solow Polasky measurement as in the previous work. This approach was proposed by Solow and Polasky in 1994 to estimate the diversity of a biological population in an eco-system ${ }^{33}$. The formula to calculate diversity was redefined to normalize the range of values from $[1, \mathrm{~m}]$ to $(0, \mathrm{~m}]$ as follows:

$$
I(A)=\frac{1}{|A|} \boldsymbol{e}^{\top} F(\boldsymbol{s})^{-1} \boldsymbol{e}
$$

where $A$ is a set of drug molecules with a size of $|A|$ equal to $m, \boldsymbol{e}$ is an $m$-vector of 1 's and $\left.F(s)=\left[f\left(d_{i j}\right)\right)\right]$ is a non-singular $m \times m$ distance matrix, in which $f\left(d_{i j}\right)$ stands for the distance function of each pair of molecule provided as follows:

$$
f(d)=e^{-\theta d_{i j}}
$$


371 here we defined the distance $d_{i j}$ of molecules $s_{i}$ and $s_{j}$ by using the Tanimoto-distance 372 with ECFP6 fingerprints as follows:

373

$$
d_{i j}=d\left(s_{i}, s_{j}\right)=1-\frac{\left|s_{i} \cap s_{j}\right|}{\left|s_{i} \cup s_{j}\right|},
$$

374 where $\left|s_{i} \cap s_{j}\right|$ represents the number of common fingerprint bits, and $\left|s_{i} \cup s_{j}\right|$ is the 375 number of union fingerprint bits. 


\section{Results and Discussion}

\section{Fragmentation of molecule}

As stated we decomposed each molecule into a series of fragments with the BRICS algorithm to construct a fragment-molecule pair. Each organic compound can be split into retrosynthetically interesting chemical substructures with a compiled elaborate set of rules. For the ChEMBL and LIGAND sets, we respectively obtained 194,782 and 2,223 fragments. We further split the LIGAND set into three parts: active ligands $\left(\right.$ LIGAND $\left.^{+}, 2,638\right)$, inactive ligands $\left(L I G A N D^{-}, 2710\right)$ and undetermined ligands $\left(L I G A N D^{0}, 5480\right)$ based on the $\mathrm{pX}$ of bioactivity for $\mathrm{A}_{2 \mathrm{~A}} \mathrm{AR}$. The number of fragments in these four datasets have a similar distribution (Fig. 3A) and there are approximately five fragments on average for each molecule with a $95 \%$ confidence between $[0,11]$ (Fig. 3A).

In the LIGAND set the three subsets have a similar molecular weight distribution of the fragments (Fig. 3B) with an average of $164.3 \mathrm{Da}$, smaller than in the ChEMBL set (247.3 Da). In order to check the similarity of these fragments we used the Tanimoto similarity calculation with ECFP4 fingerprints between each pair of fragments in the same dataset. We found that most of them were smaller than 0.5 indicating that they are dissimilar to each other (Fig. 3C). Especially, the fragments in the $L I G A N D^{+}$set have the largest diversity. Moreover, the distribution of different fragments in these three subsets of the LIGAND set are shown in Fig. 3D. The molecules in these three subsets have their unique fragments and share some common substructures. 

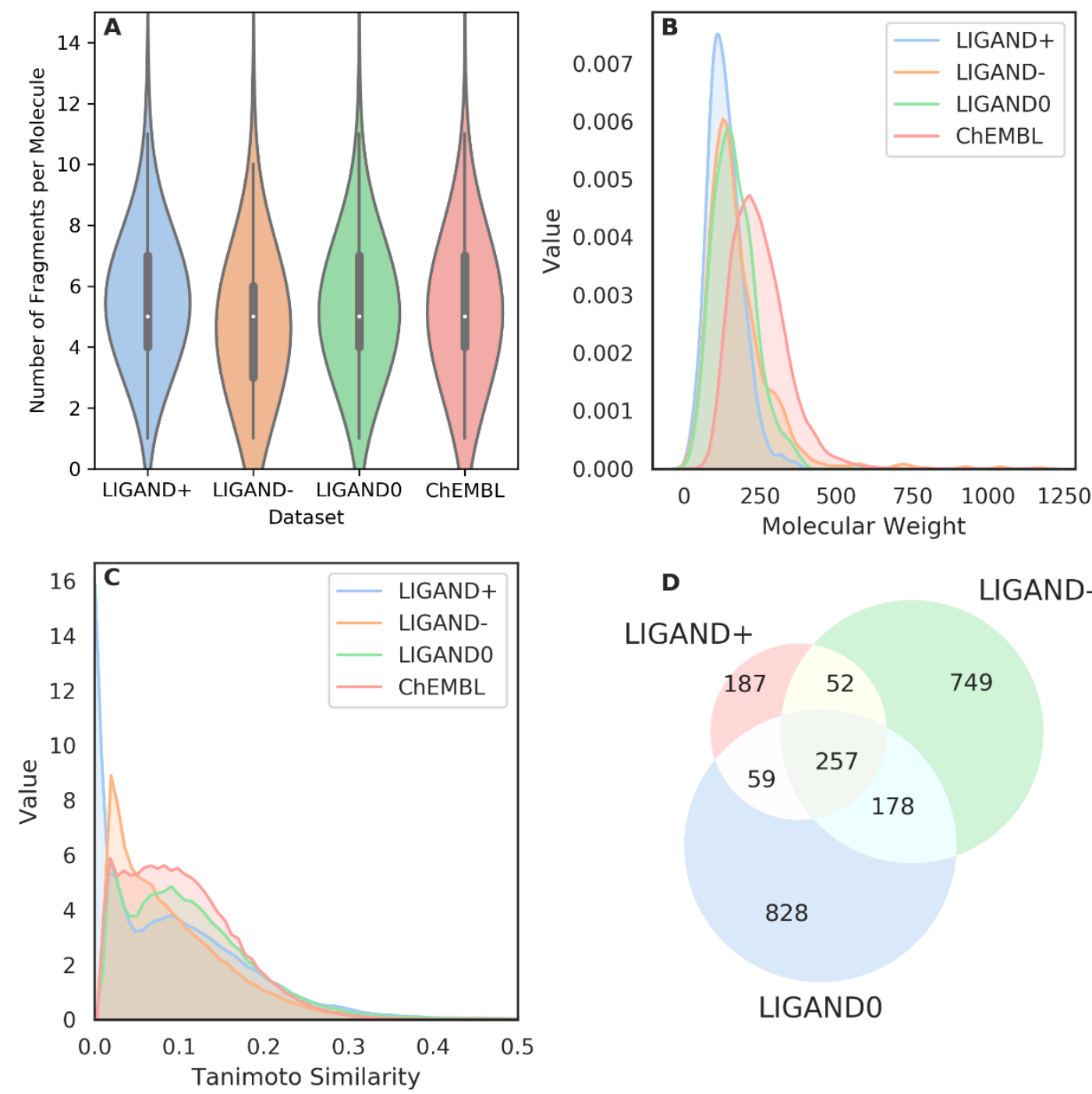

399

Fig 3: Analysis of some properties of fragments in the ChEMBL set and three LIGAND subsets.

401

(A) Violin plot for the distribution of the number of fragments per molecules; (B) Distribution of molecular weight of these fragments; (C) Distribution of the similarity of the fragments measured by the Tanimoto-similarity with ECFP4 fingerprints; (D) Venn diagram for the intersection of the fragments existing in the three subsets of the LIGAND set.

405 


\begin{tabular}{ccccccc}
\hline \multirow{2}{*}{ Methods } & \multicolumn{2}{c}{ Pre-trained Model } & \multicolumn{2}{c}{ Fine-tuned Model } & \multirow{2}{*}{ Time } & \multirow{2}{*}{ Memory } \\
\cline { 2 - 5 } & Validity & Accuracy & Validity & Accuracy & & \\
\hline $\begin{array}{c}\text { Graph } \\
\text { Transformer }\end{array}$ & $100 \%$ & $99.3 \%$ & $100 \%$ & $99.2 \%$ & $453.8 \mathrm{~s}$ & $14.5 \mathrm{~GB}$ \\
\hline $\begin{array}{c}\text { Sequential } \\
\text { Transformer }\end{array}$ & $96.7 \%$ & $72.0 \%$ & $99.3 \%$ & $87.3 \%$ & $832.3 \mathrm{~s}$ & $31.7 \mathrm{~GB}$ \\
\hline LSTM-BASE & $93.9 \%$ & $44.1 \%$ & $98.7 \%$ & $77.9 \%$ & $834.6 \mathrm{~s}$ & $5.5 \mathrm{~GB}$ \\
\hline LSTM+ATTN & $89.7 \%$ & $52.2 \%$ & $96.4 \%$ & $84.2 \%$ & $1212.5 \mathrm{~s}$ & $15.9 \mathrm{~GB}$ \\
\hline
\end{tabular}



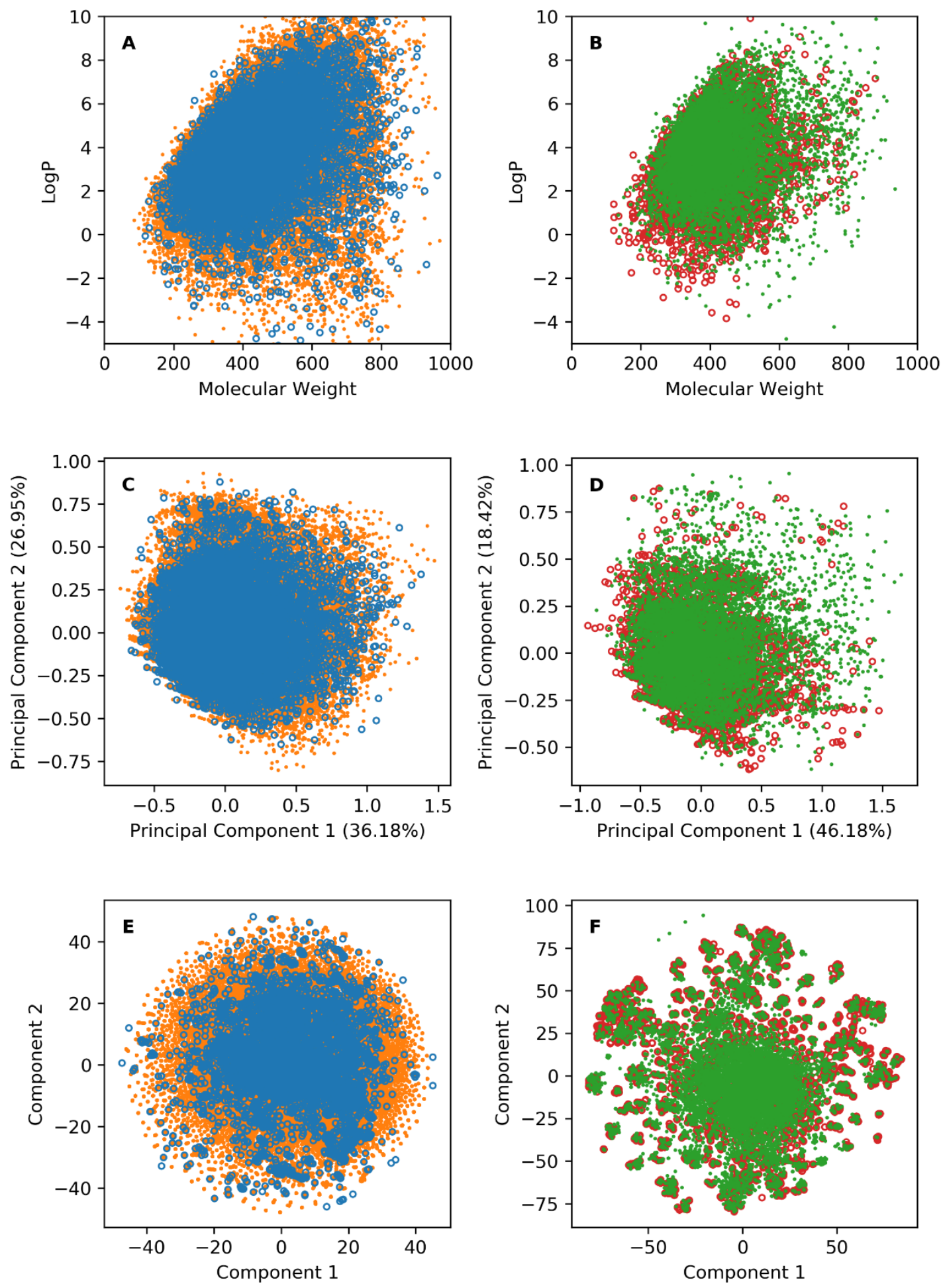

- ChEMBL Set $\circ$ Pre-trained Model $\circ$ LIGAND Set $\quad$ Fine-tuned Model

428 Fig. 4: The chemical space of generated molecules by the Graph Transformer pre-trained on the 429 ChEMBL set (A, C and E) and being fine-tuned on the LIGAND set (B, D and F). Chemical space was represented by either $\log \mathrm{P} \sim \mathrm{MW}$ (A, B) and first two components in PCA on PhysChem descriptors (C, D) and t-SNE on ECFP6 fingerprints (E, F). 
The graph representation for molecules has more advantages over the SMILES representation when dealing with fragment-based molecule design: 1) Invariance in the local scale: During the process of molecule generation, multiple fragments in a given scaffold can be put into any position in the output matrix without changing the order of atoms and bonds in that scaffold. 2) Extendibility in the global scale: When fragments in the scaffold are growing or being linked, they can be flexibly appended in the end column of the graph matrix while the original data structure does not need changing. 3) Free of grammar: Unlike in SMILES sequences there is no explicit grammar to constrain the generation of molecules, such as the parentheses for branches and the numbers for rings in SMILES; 4) Accessibility of chemical rules: For each added atom or bond the algorithm can detect if the valence of atoms is valid or not and mask invalid atoms or bonds in the vocabulary to guarantee the whole generated matrix can be successfully parsed into a molecule. With these advantages the Graph Transformer generates molecules faster while using less computational resources.

However, after examining the QED scores and SA scores we found that although the distribution of QED scores was similar between the methods (Figure 5A,C), the synthesizability of the molecules generated by the Graph Transformer were not better than the SMILES-based generators. This was especially true when fine-tuning on the LIGAND set. A possible reason is that molecules generated by the Graph Transformer contain uncommon rings when the model dealt with long-distance dependencies. In addition, because of more complicated data structure and presence of more parameters in the model, Graph Transformer did not outperform for the synthesizability of generated molecules when being trained on the small dataset (e.g. the LIGAND set). It is also worth noticing that there still was a small fraction of generated molecules that did not contain the required scaffolds which is caused by a kekulization problem. For example, a scaffold ' $\mathrm{CCC}$ ' can be grown into ' $\mathrm{C} 1=\mathrm{C}(\mathrm{C}) \mathrm{C}=\mathrm{CC}=\mathrm{C} 1$ '. After being sanitized, it can be transformed into ' $\mathrm{c} 1 \mathrm{c}(\mathrm{C}) \mathrm{cccc} 1$ '. In this process one single bond in the scaffold is changed to an aromatic bond, which causes a mismatch between the 
scaffold and the molecule. Currently our algorithm cannot solve this problem because if the aromatic bond is taken into consideration, the valence of aromatic atoms is difficult to be calculated accurately. This would lead to the generation of invalid molecules. Therefore, there is no aromatic bond provided in the vocabulary and all of the aromatic rings are inferred automatically through the molecule sanitization method in RDKit.
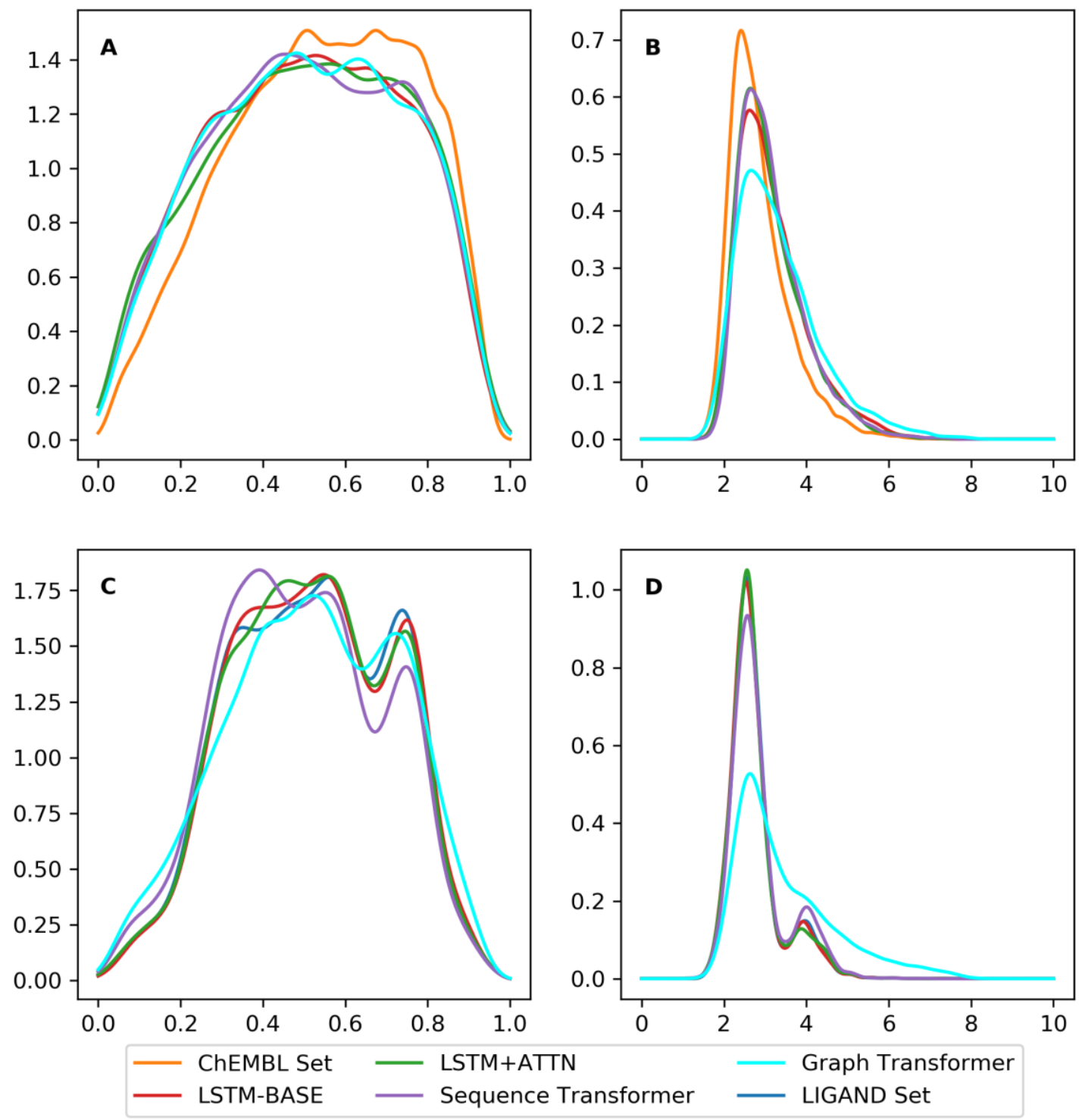

Fig. 5: the distribution of the QED score $(A, C)$ and SA score $(B, D)$ of desired ligands in the ChEMBL set and LIGAND set and of molecules generated by four different generators. For the QED score, four generators had the same performance as the molecules in both ChEMBL set (A) and the LIGAND set (C). For the SA score, Graph Transformer did not outperform three other SMILES-based generators in ChEMBL set (B) and even worse in the LIGAND set (D). 


\section{Policy gradient}

Because the Graph Transformer generates molecules accurately and fast it was chosen as the agent in the RL framework. Two objectives were tested in the training process of this work. The first one was affinity towards $\mathrm{A}_{2 \mathrm{~A}} \mathrm{AR}$, which is predicted by the random forest-based regression model from DrugEx v2; the second one was the QED score calculated with RDKit to measure how similar the generated molecule is to known approved drugs. With the policy gradient method as the reinforcement learning framework two cases were tested. On the one hand, predicted affinity for $\mathrm{A}_{2 \mathrm{~A}} \mathrm{AR}$ was considered without the QED score. On the other hand, both objectives were used to optimize the model with Pareto ranking. In the first case $86.1 \%$ of the generated molecules were predicted active, while the percentage of predicted active molecules in the second case was $74.6 \%$. Although the generator generated more active ligands without the QED score constraint most of them are not drug-like as they always have a molecular weight larger than 500Da. However, when we checked the chemical space represented by tSNE with ECFP6 fingerprints the overlap region between generated molecules and ligands in the training set was not complete implying that they fall out of the applicability domain of the regression model.

In DrugEx v2, we provided an exploration strategy which simulated the idea of evolutionary algorithms such as crossover and mutation manipulations. However, when coupled to the Graph Transformer there were some difficulties and we had to give up this strategy. Firstly, the mutation strategy did not improve with different mutation rates. A possible reason is that before being generated, part the molecule was fixed with a given scaffold counteracting the effect of mutation caused by the mutation net. Secondly, the crossover strategy is computationally very expensive in this context. This strategy needs the convergence of model training and iteratively updates the parameters in the agent. With multiple iterations, it takes a long period of time beyond the computational resources we can currently access. As a result, we updated the exploration strategy as mentioned in the Methods section with six different exploration 
rates: $[0.0,0.1,0.2,0.3,0.4,0.5]$.

Table 2: the performance of the Graph Transformer with different exploration rates in the RL

framework.

\begin{tabular}{ccccc}
\hline $\boldsymbol{\varepsilon}$ & Accuracy & Desirability & Uniqueness & Diversity \\
\hline $\mathbf{0 . 0}$ & $99.7 \%$ & $74.6 \%$ & $60.7 \%$ & 0.879 \\
\hline $\mathbf{0 . 1}$ & $99.7 \%$ & $66.8 \%$ & $75.0 \%$ & 0.842 \\
\hline $\mathbf{0 . 2}$ & $99.8 \%$ & $61.6 \%$ & $80.2 \%$ & 0.879 \\
\hline $\mathbf{0 . 3}$ & $99.7 \%$ & $56.8 \%$ & $89.8 \%$ & 0.874 \\
$\mathbf{0 . 4}$ & $99.7 \%$ & $54.8 \%$ & $88.8 \%$ & 0.859 \\
\hline $\mathbf{0 . 5}$ & $99.7 \%$ & $46.8 \%$ & $88.5 \%$ & 0.875 \\
\hline
\end{tabular}

Changes to the exploration rate do not influence accuracy and have a low effect on diversity.

However, desirability (finding active ligands) and uniqueness can be influenced significantly.

Empirically determining an optimal value for a given chemical space is recommended.

After training of the models, multiple scaffolds were input 10 times to generate molecules. The results for accuracy, desirability, uniqueness, and diversity with different exploration rates are shown in Table 2. With a low $\varepsilon$ the model generates more desired molecules, but the uniqueness of the generated molecules can be improved. At $\varepsilon=0.3$ the model generated the highest percentage of unique desired molecules $(56.8 \%)$.

518 Diversity was always larger than 0.84 and the model achieved the largest value $(0.88)$

519 with $\varepsilon=0.0$ or $\varepsilon=0.2$. The chemical space represented by tSNE with ECFP6 520 fingerprints confirms that our exploration strategy produces a set of generated 521 molecules completely covering the region occupied by the LIGAND set (Fig. 6). 

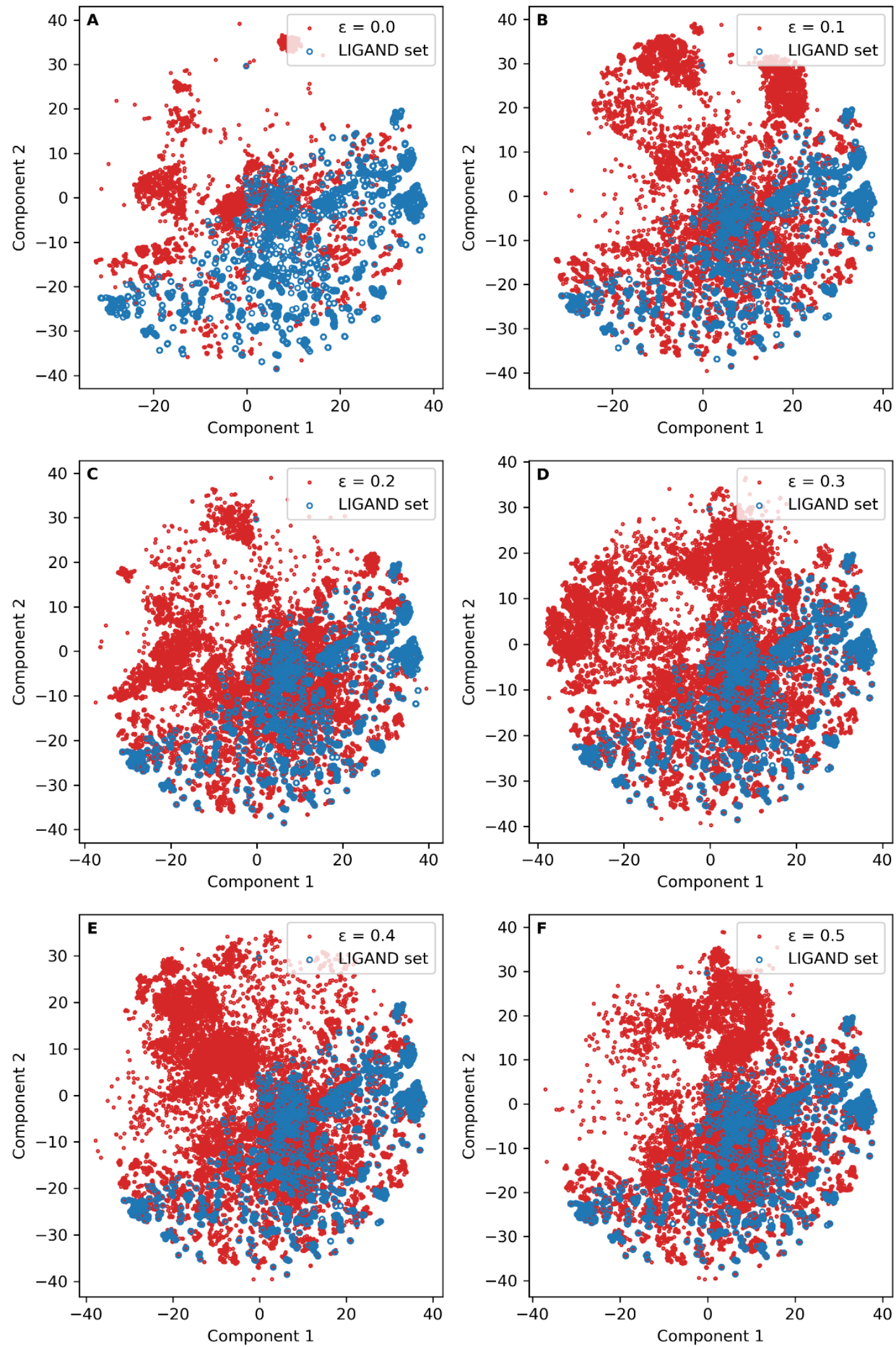

Fig. 6: The chemical space of generated molecules by the Graph Transformer trained with different exploration rates in the RL framework. The chemical space was represented by t-SNE on ECFP6 fingerprints. 


\section{Generated Molecules}

529 In the chemical space making up antagonists of $A_{2 A} A R$ there are several well-known scaffolds. Examples include furan, triazine, aminotriazole, and purine derivatives such as xanthine and azapurine. The Graph Transformer model produced active ligands for

532

533 $\mathrm{A}_{2 \mathrm{~A}} \mathrm{AR}$ (inferred from the predictors) with different combinations of these fragments as scaffolds. Taking these molecules generated by the Graph Transformer as an example, we filtered out the molecules with potentially reactive groups (such as aldehydes) and uncommon ring systems and listed 30 desired molecules as putative $\mathrm{A}_{2 \mathrm{~A}} \mathrm{AR}$ ligands/antagonists (Fig. 7). For each scaffold five molecules were selected and assigned in the same row. These molecules are considered a valid starting point for further considerations and work (e.g. molecular docking or simulation).

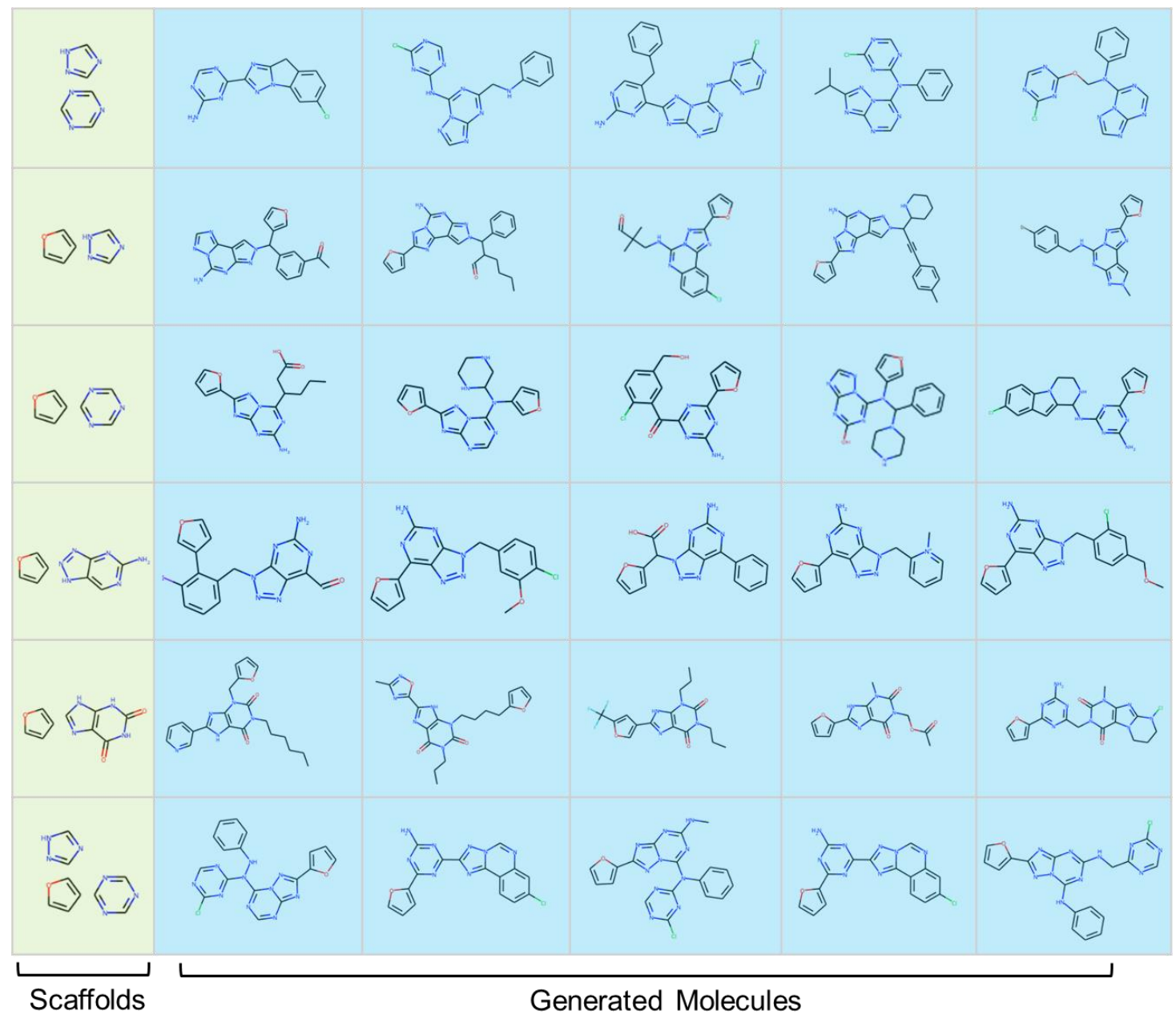

Fig. 7: Sample of generated molecules with the Graph Transformer with different scaffolds. 
These scaffolds include: furan, triazine, aminotriazole, xanthine, and azapurine. The generated molecules based on the same scaffolds are aligned in the same row.

\section{Conclusions and Future Perspectives}

In this study, DrugEx was updated with the ability to design novel molecules based on scaffolds consisting of multiple fragments as input. In this version ( $v 3)$, a new positional encoding scheme for atoms and bonds was proposed to make the Transformer model deal with a molecular graph representation. With one model, multiple fragments in a given scaffold can be grown at the same time and connected to generate a new molecule. In addition, chemical rules on valence are enforced at each step of the process of molecule generation to ensure that all generated molecules are valid. These advantages are impossible to be embodied in SMILES-based generation, as SMILES-based molecules are constrained by grammar that allows a $2 \mathrm{D}$ topology to be represented in a sequential way. With multi-objective reinforcement learning the model generates drug-like ligands, in our case for the $\mathrm{A}_{2 \mathrm{~A}} \mathrm{AR}$ target.

In future work, the Graph Transformer will be extended to include other information as input to design drugs conditionally. For example, proteochemometric modelling (PCM) can take information for both ligands and targets as input to predict the affinity of their interactions, which allows generation of compounds that are promiscuous (useful for e.g., viral mutants) or selective (useful for e.g., kinase inhibitors) ${ }^{34}$. The Transformer can then be used to construct inverse PCM models which take the protein information as input (e.g. sequences, structures, or descriptors) to design active ligands for a given protein target without known ligands. Moreover, the Transformer can also be used for lead optimization. For instance, the input can be a "hit" already, generating "optimized" ligands, or a "lead" with side effects to produce ligands with a better ADME/tox profile.

\section{Authors' Contributions}

XL and GJPvW conceived the study and performed the experimental work and analysis. 
571 KY, APIJ nd HWTvV provided feedback and critical input. All authors read,

572 commented on and approved the final manuscript.

573

\section{Acknowledgements}

575 XL thanks Chinese Scholarship Council (CSC) for funding, GJPvW thanks the Dutch

576 Research Council and Stichting Technologie Wetenschappen (STW) for financial 577 support (STW-Veni \#14410). Thanks go to Dr. Xue Yang for verifying Table S1 and Dr.

578 Anthe Janssen checking the convergence of t-SNE. We also acknowledge Bert 579 Beerkens for providing the common scaffolds used to generate molecules as an example.

580

581 Competing Interests

582 The authors declare that they have no competing interests 


\section{References}

1. P. G. Polishchuk, T. I. Madzhidov and A. Varnek, J Comput Aided Mol Des, 2013, 27, 675-679.

2. P. J. Hajduk and J. Greer, Nat Rev Drug Discov, 2007, 6, 211-219.

3. G. L. Card, L. Blasdel, B. P. England, C. Zhang, Y. Suzuki, S. Gillette, D. Fong, P. N. Ibrahim, D. R. Artis, G. Bollag, M. V. Milburn, S. H. Kim, J. Schlessinger and K. Y. Zhang, Nat Biotechnol, 2005, 23, 201-207.

4. Y. Bian and X. S. Xie, AAPS J, 2018, 20, 59.

5. J. P. Hughes, S. Rees, S. B. Kalindjian and K. L. Philpott, Br J Pharmacol, 2011, 162, 1239-1249.

6. C. Sheng and W. Zhang, Med Res Rev, 2013, 33, 554-598.

7. R. Santos, O. Ursu, A. Gaulton, A. P. Bento, R. S. Donadi, C. G. Bologa, A. Karlsson, B. Al-Lazikani, A. Hersey, T. I. Oprea and J. P. Overington, Nat Rev Drug Discov, 2017, 16, 19-34.

8. B. B. Fredholm, Exp Cell Res, 2010, 316, 1284-1288.

9. J. F. Chen, H. K. Eltzschig and B. B. Fredholm, Nat Rev Drug Discov, 2013, 12, 265-286.

10. S. Moro, Z. G. Gao, K. A. Jacobson and G. Spalluto, Med Res Rev, 2006, 26, 131-159.

11. W. Jespers, A. Oliveira, R. Prieto-Diaz, M. Majellaro, J. Aqvist, E. Sotelo and H. Gutierrez-deTeran, Molecules, 2017, 22.

12. X. Liu, A. P. IJzerman and G. J. P. van Westen, Methods Mol Biol, 2021, 2190, 139-165.

13. Y. LeCun, Y. Bengio and G. Hinton, Nature, 2015, 521, 436-444.

14. R. Gomez-Bombarelli, J. N. Wei, D. Duvenaud, J. M. Hernandez-Lobato, B. Sanchez-Lengeling, D. Sheberla, J. Aguilera-Iparraguirre, T. D. Hirzel, R. P. Adams and A. Aspuru-Guzik, ACS Cent Sci, 2018, 4, 268-276.

15. M. H. S. Segler, T. Kogej, C. Tyrchan and M. P. Waller, ACS Cent Sci, 2018, 4, 120-131.

16. S.-L. Benjamin, O. Carlos, G. Gabriel L. and A.-G. Alan, Optimizing distributions over molecular space. An Objective-Reinforced Generative Adversarial Network for Inverse-design Chemistry (ORGANIC), 2017.

17. M. Olivecrona, T. Blaschke, O. Engkvist and H. Chen, Journal of cheminformatics, 2017, 9, 48.

18. T. Blaschke, J. Arus-Pous, H. Chen, C. Margreitter, C. Tyrchan, O. Engkvist, K. Papadopoulos and A. Patronov, Journal of chemical information and modeling, 2020, 60, 5918-5922.

19. J. Lim, S. Y. Hwang, S. Moon, S. Kim and W. Y. Kim, Chem Sci, 2019, 11, 1153-1164.

20. Y. Li, J. Hu, Y. Wang, J. Zhou, L. Zhang and Z. Liu, Journal of chemical information and modeling, 2020, 60, 77-91.

21. J. Arus-Pous, A. Patronov, E. J. Bjerrum, C. Tyrchan, J. L. Reymond, H. Chen and O. Engkvist, Journal of cheminformatics, 2020, 12, 38.

22. A. Vaswani, N. Shazeer, N. Parmar, J. Uszkoreit, L. Jones, A. N. Gomez, L. Kaiser and I. J. a. e.-p. Polosukhin, Journal, 2017, arXiv:1706.03762.

23. Y. Yang, S. Zheng, S. Su, C. Zhao, J. Xu and H. Chen, Chem Sci, 2020, 11, 8312-8322.

24. X. Liu, K. Ye, H. W. T. van Vlijmen, A. P. IJzerman and G. J. P. van Westen, Journal of cheminformatics, 2019, 11, 35.

25. X. Liu, K. Ye, H. W. T. van Vlijmen, M. T. M. Emmerich, I. A. P. and G. J. P. van Westen, Journal of cheminformatics, 2021, 13, 85.

26. A. Gaulton, L. J. Bellis, A. P. Bento, J. Chambers, M. Davies, A. Hersey, Y. Light, S. McGlinchey, D. Michalovich, B. Al-Lazikani and J. P. Overington, Nucleic Acids Res, 2012, 40, D1100-1107.

27. J. Degen, C. Wegscheid-Gerlach, A. Zaliani and M. Rarey, ChemMedChem, 2008, 3, 1503-1507. 
28. PyTorch, https://pytorch.org/).

29. A. Dosovitskiy, L. Beyer, A. Kolesnikov, D. Weissenborn, X. Zhai, T. Unterthiner, M. Dehghani, M. Minderer, G. Heigold, S. Gelly, J. Uszkoreit and N. J. a. e.-p. Houlsby, Journal, 2020, arXiv:2010.11929.

630 30. G. R. Bickerton, G. V. Paolini, J. Besnard, S. Muresan and A. L. Hopkins, Nat Chem, 2012, 4, 90-

631 98.

632 31. Scikit-Learn: machine learning in Python, http://www.scikit-learn.org/).

633 32. P. Ertl and A. Schuffenhauer, Journal of cheminformatics, 2009, 1, 8.

634 33. A. R. Solow and S. Polasky, Environmental and Ecological Statistics, 1994, 1, 95-103.

635 34. G. J. van Westen, J. K. Wegner, P. Geluykens, L. Kwanten, I. Vereycken, A. Peeters, A. P. Ijzerman, 636 H. W. van Vlijmen and A. Bender, PLoS One, 2011, 6, e27518.

637 
Table S1: Atoms in vocabulary for graph-based molecule generation. The column of "Symbol" is the symbol of the atom and its charge; the column of "Valence" is the value of valence of the state of each chemical element; the "Number" column stands for the index of each element in the periodic

641 table, the last row is the unique word for each state of these elements, a combination of its valence 642 and symbol.

\begin{tabular}{|c|c|c|c|c|}
\hline Symbol & Valence & Charge & Number & Word \\
\hline $\mathrm{O}$ & 2 & 0 & 8 & $2 \mathrm{O}$ \\
\hline $\mathrm{O}+$ & 3 & 1 & 8 & $3 \mathrm{O}+$ \\
\hline O- & 1 & -1 & 8 & $10-$ \\
\hline $\mathrm{C}$ & 4 & 0 & 6 & $4 \mathrm{C}$ \\
\hline $\mathrm{C}+$ & 3 & 1 & 6 & $3 \mathrm{C}+$ \\
\hline C- & 3 & -1 & 6 & $3 C-$ \\
\hline $\mathrm{N}$ & 3 & 0 & 7 & $3 \mathrm{~N}$ \\
\hline $\mathrm{N}+$ & 4 & 1 & 7 & $4 \mathrm{~N}+$ \\
\hline N- & 2 & -1 & 7 & $2 \mathrm{~N}-$ \\
\hline $\mathrm{Cl}$ & 1 & 0 & 17 & $1 \mathrm{Cl}$ \\
\hline S & 2 & 0 & 16 & $2 \mathrm{~S}$ \\
\hline S & 6 & 0 & 16 & $6 \mathrm{~S}$ \\
\hline S & 4 & 0 & 16 & $4 \mathrm{~S}$ \\
\hline $\mathrm{S}+$ & 3 & 1 & 16 & $3 \mathrm{~S}+$ \\
\hline S+ & 5 & 1 & 16 & $5 \mathrm{~S}+$ \\
\hline S- & 1 & -1 & 16 & $1 \mathrm{~S}-$ \\
\hline $\mathrm{F}$ & 1 & 0 & 9 & $1 \mathrm{~F}$ \\
\hline I & 1 & 0 & 53 & $1 \mathrm{I}$ \\
\hline I & 5 & 0 & 53 & $5 \mathrm{I}$ \\
\hline I+ & 2 & 1 & 53 & $2 \mathrm{I}+$ \\
\hline $\mathrm{Br}$ & 1 & 0 & 35 & $1 \mathrm{Br}$ \\
\hline $\mathrm{P}$ & 5 & 0 & 15 & $5 \mathrm{P}$ \\
\hline $\mathrm{P}$ & 3 & 0 & 15 & $3 \mathrm{P}$ \\
\hline $\mathrm{P}+$ & 4 & 1 & 15 & $4 \mathrm{P}+$ \\
\hline $\mathrm{Se}$ & 2 & 0 & 34 & $2 \mathrm{Se}$ \\
\hline $\mathrm{Se}$ & 6 & 0 & 34 & $6 \mathrm{Se}$ \\
\hline $\mathrm{Se}$ & 4 & 0 & 34 & $4 \mathrm{Se}$ \\
\hline $\mathrm{Se}+$ & 3 & 1 & 34 & $3 \mathrm{Se}+$ \\
\hline $\mathrm{Si}$ & 4 & 0 & 14 & $4 \mathrm{Si}$ \\
\hline B & 3 & 0 & 5 & $3 B$ \\
\hline B- & 4 & -1 & 5 & $4 \mathrm{~B}-$ \\
\hline As & 5 & 0 & 33 & $5 \mathrm{As}$ \\
\hline As & 3 & 0 & 33 & $3 \mathrm{As}$ \\
\hline As+ & 4 & 1 & 33 & $4 \mathrm{As}+$ \\
\hline $\mathrm{Te}$ & 2 & 0 & 52 & $2 \mathrm{Te}$ \\
\hline $\mathrm{Te}$ & 4 & 0 & 52 & $4 \mathrm{Te}$ \\
\hline $\mathrm{Te}+$ & 3 & 1 & 52 & $3 \mathrm{Te}+$ \\
\hline$*$ & 0 & 0 & 0 & $*$ \\
\hline
\end{tabular}


Algorithm encoding:

Input:

mol: structure of the kekulized molecule

subs: structure of the scaffolds

vocab: vocabulary of tokens which is consisted of graph matrix

Output :

matrix: the $\mathrm{n} \times 5$ matrix to represents the molecular graph.

\# Ensure the atom of the subs are put at the start in the molecule

mol $\leftarrow$ RANK_ATOM_BY_SUB (mol, subs)

sub_atoms $\leftarrow$ GET_ATOMS (subs)

sub_bonds $\leftarrow$ GET_BONDS (subs)

mol_atoms $\leftarrow$ GET_ATOMS (mol)

frag, grow, link $\leftarrow\left[\left({ }^{G O} ', 0,0,0,1\right)\right],[],[(0,0,0,0,0)]$

For atom in mol_atoms:

\# The bonds which connect to the atom having the index before this atom bonds $\leftarrow$ GET_LEFT_BONDS (mol, atom)

For bond in bonds:

tk_bond $\leftarrow$ GET_TOKEN (vocab, bond)

other $\leftarrow$ GET_OTHER_ATOM $(\mathrm{mol}$, atom, bond $)$

If IS_FIRST (bonds, bond):

tk_atom $\leftarrow$ GET_TOKEN (vocab, atom)

Else:

tk_atom $\leftarrow$ GET_TOKEN (vocab, None)

\# The index of the scaffold in which the current atom locates

\# Its value starts from 1 . If it is not in the scaffold, it will be 0 scf $\leftarrow$ GET_FRAG_ID (subs, atom)

column $\leftarrow$ (tk_atom, tk_bond, GET_INDEX (other), GET_INDEX (atom), scf)

If other in sub_atoms and atom in sub_atoms and bond not in sub_bonds:

Insert column to link

Else if bond in sub_bonds:

Insert column to frag

Else:

Insert column to grow

End

End

Insert ('EOS', $\theta, \theta, \theta, \theta$ ) to grow

matrix $\leftarrow$ CONCATENATE_BY_COLUMN (frag, grow, link)

Return matrix 


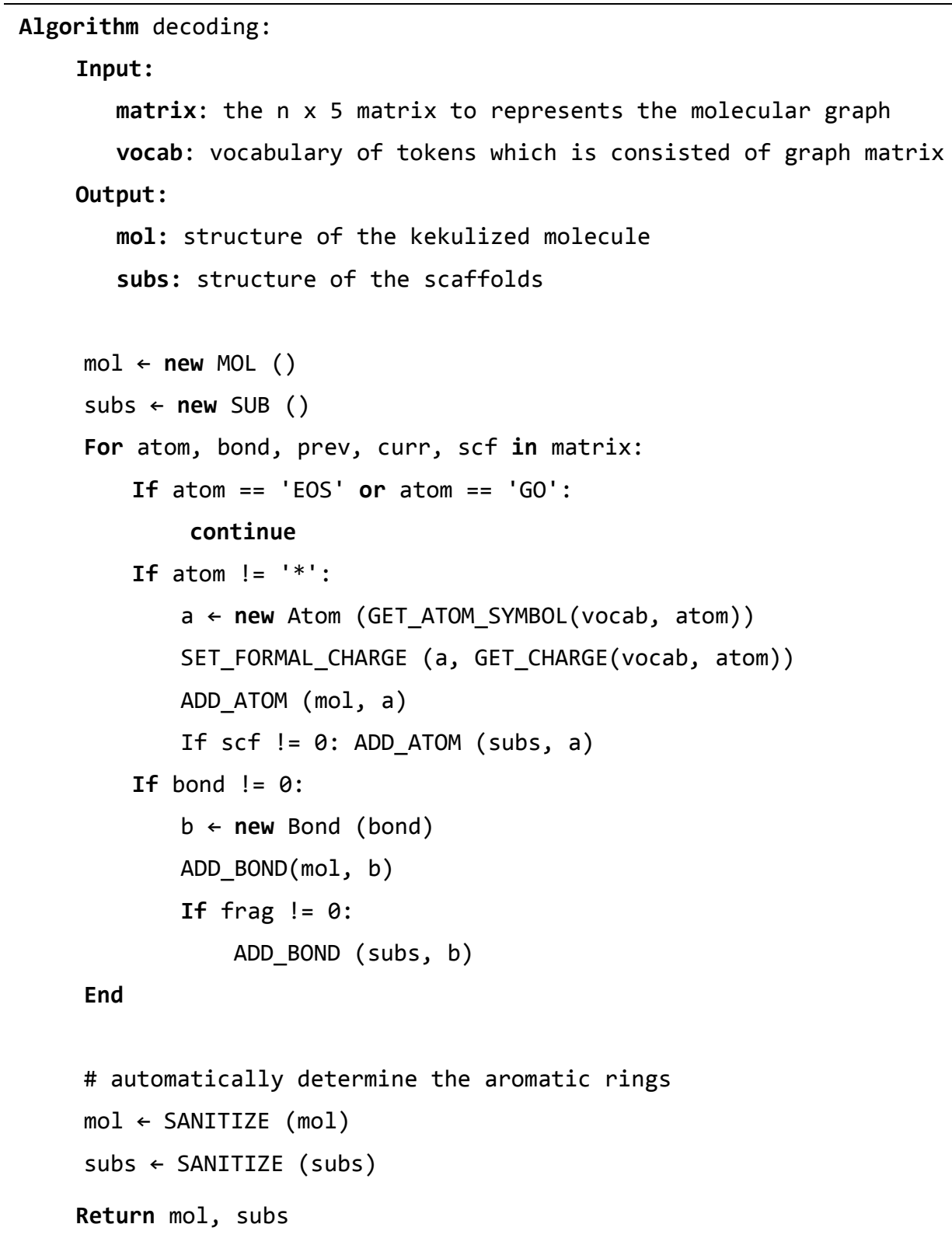

